\title{
APLICAÇÃO DE MÉTODOS GEOFíSICOS PARA IDENTIFICAR ÁREAS CONTAMINADAS POR RESÍDUOS DE UM CURTUME
}

\author{
Sergio Junior da Silva Fachin ${ }^{1}$, Felix Huber², Shozo Shiraiwa ${ }^{3}$, \\ Welitom Rodrigues Borges ${ }^{4}$ e Renato Blat Migliorini ${ }^{5}$ \\ Recebido em 16 agosto, 2005 / Aceito em 21 março, 2006 \\ Received on August 16, 2005 / Accepted on March 21, 2006
}

\begin{abstract}
The main problem on environmental investigations is the determination of contaminated extension area. This work exhibit some results obtained with the geophysical methods applied to locate potentially contaminated areas with tanning residues. The area is located near to the city of Várzea Grande, Mato Grosso State. It was now interdicted and disabled for the residues deposition. The data were acquired from electrical profiles, with inline dipole-dipole array and $4 \mathrm{~m}$ spacing electrodes, allowing 5 investigation depths. The data of apparent electric conductivity were acquired in 2 dipole modes (horizontal and vertical coplanar) with intercoil spacing of 10 meters. The $200 \mathrm{MHz}$ unshielded antennas were used to collect GPR data. The station interval to each 0.25 meters and 128 stacks. The areas were classified in Contaminated Area - AC - $\left(\sigma_{a}>40 \mathrm{mS} / \mathrm{m}, \rho<30 \mathrm{ohm} . \mathrm{m}\right.$ and high attenuation of GPR), Suspect Area - AS - ( $\sigma_{a}$ of 20 to $40 \mathrm{mS} / \mathrm{m}, \rho$ of 30 to 50 ohm.m) and Without Contaminated - AN - $\left(\sigma_{a}<20 \mathrm{mS} / \mathrm{m}, \rho>50 \mathrm{ohm} . \mathrm{m}\right.$ and low attenuation of GPR). The integrated results allow the contamination maps elaboration, indicating a potentially contaminated area with a preferential NW-SE flux. The geophysics and geological information showed an efficient preliminary analysis in the identification of contaminated areas.
\end{abstract}

Keywords: Contamination, industrial residues, tanning, EM-34, resistivity, GPR, Várzea Grande.

RESUMO. Um dos problemas existentes em investigações ambientais é a determinação da extensão do contaminante no solo. Este trabalho mostra alguns resultados obtidos com os métodos eletromagnético indutivo, eletrorresistividade e georadar (GPR - Ground Penetrating Radar) aplicados para determinar e localizar áreas potencialmente contaminadas com resíduos industriais de um curtume. A área está localizada na cidade de Várzea Grande - MT, e atualmente está interditada e desativada para a deposição de resíduos. A aquisição de dados consistiu em perfis de caminhamento elétrico, caminhamento eletromagnético indutivo e perfis common offset de GPR. No caminhamento elétrico foi utilizado o arranjo dipolo-dipolo, com espaçamento entre os dipolos de 4 metros e 5 níveis de investigação. Os dados de condutividade elétrica aparente foram adquiridos com o equipamento EM-34, nos modos dipolo magnético horizontal e vertical a cada 2 metros, com espaçamento entre as bobinas de 10 metros. Nas aquisições de GPR foram utilizadas antenas não-blindadas de $200 \mathrm{MHz}$, empilhamento de 128 sinais e traços coletados a cada 0,25 metros. Após as coletas dos dados, foram realizadas as classificações e delimitações das áreas de acordo com o padrão das anomalias de cada método. As áreas foram classificadas em Áreas Contaminadas - AC ( $\sigma_{a}>40 \mathrm{mS} / \mathrm{m}, \rho<30 \mathrm{ohm} . \mathrm{m}$ e alta atenuação do sinal do georadar), Áreas Suspeitas de Contaminação $-\mathrm{AS}$ ( $\sigma_{a}$ de 20 a $40 \mathrm{mS} / \mathrm{m}, \rho$ de 30 a 50 ohm.m) e Áreas Não-Contaminadas - AN ( $\sigma_{a}<20 \mathrm{mS} / \mathrm{m}, \rho>50 \mathrm{ohm} . \mathrm{m}$ e baixa atenuação do sinal do GPR). Os resultados integrados possibilitaram a elaboração de mapas de contaminação, indicando uma área potencialmente contaminada com um sentido preferencial NW-SE. As informações geofísicas aliadas à informação geológica de superfície e de poço mostraram uma análise preliminar eficiente na identificação de áreas potencialmente contaminadas.

Palavras-chave: Contaminação, resíduos industriais, curtume, EM-34, eletrorresistividade, GPR, Várzea Grande-MT.

${ }^{1}$ Curso de Pós-Graduação em Geofísica. Instituto de Astronomia, Geofísica e Ciências Atmosféricas, Rua do Matão, 1226, 05508-090 Cidade Universitária, São Paulo, SP, Brasil. Fone: (11) 3091-2792 - E-mail: fachin@iag.usp.br

${ }^{2}$ Av. Cuiabá, n 596, Bairro Cidade Alta. 78020-413 Cuiabá, MT, Brasil. Fone: (65) 637-4496 - E-mail: 77felix@gmail.com

${ }^{3}$ Departamento de Física, Universidade Federal de Mato Grosso, Avenida Fernando Correia da Costa, s/n-Bloco F / sala 224 - ICET. 78060-900 Cuiabá, MT, Brasil. Tel: (65) 615-8745/8751; Fax: (65) 615-8764 - E-mail: shozo@cpd.ufmt.br

${ }^{4}$ Curso de Pós-Graduação em Geofísica. Instituto de Astronomia, Geofísica e Ciências Atmosféricas, Rua do Matão, 1226, Cidade Universitária, 05508-090 São Paulo, SP, Brasil. Fone: (11) 3091-2792 - E-mail: welitom@iag.usp.br

${ }^{5}$ Departamento de Geologia Geral, Universidade Federal de Mato Grosso, Avenida Fernando Correia da Costa, s/n. ICET, Bloco D, sala 108. 78060-900 Cuiabá, MT, Brasil. Tel: (065) 615-8751; Fax: (065) 615-8752 - E-mail: rbmi@terra.com.br 


\section{INTRODUÇÃO}

A atividade industrial relacionada a curtumes no Estado de Mato Grosso se mostra intensa devido ao seu enorme rebanho bovino e é de grande importância. No processo de curtimento de peles, a disposição dos resíduos no solo constitui uma prática de alto risco, devido ao acumulo e concentração de uma grande quantidade de resíduos, proveniente de uma solução, composta por água, sais de cromo, zircônio, alumínio, ferro, curtentes vegetais, sintéticos, aldeídos e parafinas sulfocloradas. Posteriormente esta solução é encaminhada para tanques de decantação, a água é encaminhada para tanques para ser tratada e voltar para 0 circuito, e o resíduo sólido precipitado, denominado de lodo, deve ser acondicionado em recipientes, ir para incineradores ou ser guardado indefinidamente em condições ótimas, ao abrigo de intempéries, insolação e outros agentes que possam romper o recipiente e espalhar o resíduo na natureza (Giannetti et al., 2001). Os altos custos, decorrentes do armazenamento e/ou da incineração, induzem a comportamentos inadequados.

Os resíduos sólidos dos curtumes são essencialmente resíduos orgânicos: pêlos, gorduras, restos de carne e lodos do tratamento biológico. 0 problema específico dos curtumes é a contaminação destes resíduos com cromo sendo que, a ocorrência da parte orgânica em conjunto com a parte inorgânica tóxica, constitui a dificuldade central do tratamento dos resíduos (Keller et al., 1997).

Segundo Chernicharo et al. (1996), nos efluentes de curtume, obtém-se, por tonelada de pele crua, cerca de $100 \mathrm{~kg}$ de resíduos sólidos contendo cerca de $4 \mathrm{~kg}$ de cromo.

De acordo com a Agencia de Proteção Ambiental dos Estados Unidos (U.S. EPA, 1997) em uma pesquisa em 1000 locais contaminados, verificou-se que o cromo é o segundo metal mais poluidor do solo e da água subterrânea, onde as principais fontes de contaminação provêm de indústrias de galvanização, manufatura têxtil, curtumes, manufatura de tintas e indústrias de preservação de madeira.

0 problema mais significante associado com 0 curtume é a contaminação de solos e água subterrânea pelo cromo hexavalente que é severamente tóxico, mutagênico, e carcinogênico no meio ambiente (Papp, 1994). Ele é também muito solúvel, móvel, e se movimenta na mesma velocidade que a água subterrânea. Ao contrário, 0 cromo trivalente é relativamente de baixa toxicidade e é imóvel em ambientes moderavelmente alcalinos e levemente ácidos.

No diagnóstico ambiental de áreas contaminadas, a realização de levantamentos geofísicos tem por objetivo básico a identificação da presença da contaminação subterrânea, além da definição das feições geológicas e hidrogeológicas dos locais investigados (CETESB, 2001). Os métodos geofísicos mais eficientes na identificação de contaminantes em subsuperfície são os métodos da eletrorresistividade, eletromagnético indutivo e 0 georadar (GPR) (Lima et al., 1995; Elis, 1999; Aquino, 2000; Shiraiwa et al., 2002; Lago, 2004; Porsani et al., 2004). Além disto, são de rápida aplicação e baixo custo financeiro, quando comparados com os demais métodos.

\section{CARACTERIZAÇÃO DA ÁREA EM ESTUDO}

A área deste trabalho está localizada próximo ao limite da área urbana da cidade de Várzea Grande-MT. As investigações diretas e indiretas foram executadas em uma área de aproximadamente 4 hectares (Figura 1). Segundo Lannes (2002) o local serviu como área de disposição de resíduos sólidos industriais provenientes de um curtume por um período ininterrupto de 10 anos. Os resíduos eram dispostos em valas com dimensões de $50 \mathrm{~m} \times 2,5 \mathrm{~m} \times 2,5 \mathrm{~m}$, e impermeabilizadas com lonas plásticas de $0,3 \mathrm{~mm}$ de espessura. No local parte destas valas foram visualmente identificadas, devido à infiltração de água de chuva e consequentemente processo de ravinamento em alguns pontos de concentração e escoamento da água pluvial. Observou-se também a disposição de resíduos em superfície, ausente de qualquer proteção, estando em contato direto com o solo e sujeito às intempéries. Contudo durante o levantamento não se notou a disposição geométrica das valas e sua exata localização devido à vegetação que cresceu sobre as camadas de aterro.

A área está situada no contexto geológico dos metassedimentos do Grupo Cuiabá, representada localmente, de acordo com Migliorini (1999), pela Formação Rio Coxipó, que é caracterizada pela presença de metadiamictitos ora de matriz arenosa e ora de matriz argilosa, possuindo clastos de quartzo, feldspatos e fragmentos de quartzitos.

Localmente, através de descrição de trincheiras obtidas por Lannes (2002), ocorrem solos concrecionários, pouco profundos, caracterizados por textura argilo-arenosa, presença de níveis de cascalhos pouco espessos com seixos de quartzo arredondados, facetados e presença de couraça ferruginosa pouco espessa sendo notada somente no leito de uma drenagem nas proximidades da área.

\section{METODOLOGIA}

Os métodos geofísicos utilizados para a caracterização desta área foram o método da eletrorresistividade, o método eletromagnético indutivo e 0 georadar (GPR). 


\section{Método da Eletrorresistividade}

A eletrorresistividade é um método baseado na determinação da resistividade elétrica dos materiais, caracterizada pela dificuldade que um determinado material impõe à passagem de correntes elétricas. Os principais fatores que influenciam na variação da resistividade elétrica dos materiais são: composição mineralógica, quantidade de água presente nos poros, sólidos totais dissolvidos (STD), porosidade e temperatura. Como resíduos contaminantes inorgânicos aumentam a quantidade de STD no solo (Elis, 1999), a área contaminada poderá ser mapeada através do contraste da resistividade elétrica do meio contaminado com o livre de contaminação. A medida de resistividade elétrica no solo é comumente feita através da injeção de corrente elétrica por um par de eletrodos de corrente, e a variação do potencial elétrico no solo é medido através de outro par de eletrodos metálicos ou um par de eletrodos não polarizáveis.

As investigações elétricas neste trabalho utilizaram a técnica do caminhamento elétrico com 0 arranjo dipolo-dipolo com espaçamento entre os dipolos de 4 metros e foram investigados 5 níveis de profundidade. Foram adquiridas 3 seções de caminhamento elétrico na área (Linhas 1, 3 e 4, Figura 1). Os dados de eletrorresistividade 2-D foram modelados no software Res2dinv (Loke, 2004), que determina automaticamente um modelo de resistividade bidimensional para o meio, a partir dos dados elétricos de campo.

\section{Método Eletromagnético Indutivo}

0 sistema eletromagnético mais comum utilizado em investigações geofísicas de engenharia e ambiental é o método de bobina dupla de fonte móvel (moving-source dual-coil method) (Mcneill, 1980). Duas bobinas separadas conectadas por um cabo de referência constituem a base do sistema. Uma bobina transmissora é utilizada para gerar um campo magnético primário e outra atua como receptor que mede tanto 0 campo primário quanto 0 campo secundário. A separação entre as bobinas é mantida fixa e o par de bobinas é movido em intervalos discretos ao longo de um perfil. 0 ponto de referência para a medida é 0 centro entre as bobinas (Reynolds, 1997). Os sistemas podem medir a componente quadratura ou em-fase (in-phase). As medidas são realizadas com as bobinas no plano vertical (modo dipolo magnético horizontal - DMH) e com as bobinas no plano horizontal (modo dipolo magnético vertical - DMV). As profundidades efetivas de investigação para os dois modos em um meio homogêneo, são respectivamente 0,75 e 1,5 vezes 0 espaçamento entre as bobinas (Mcneill, 1980). Foi utilizado neste caso um condutivímetro de solo (EM-34 da Geonics Ltd.) que fornece uma leitura direta da componente quadratura convertido para condutividade aparente $\left(\sigma_{a}\right)$ em milisiemens por metro $(\mathrm{mS} / \mathrm{m})$. Foi utilizado o cabo de separação entre as bobinas de 10 metros. As aquisições em campo foram realizadas através de caminhamento na direção de cada perfil (Linhas 4, 5, 6 e 7, Figura 1), onde a cada 2 metros foram efetuadas as leituras de condutividade aparente nos modos DMH e DMV.

Os dados de condutividade obtidos em campo foram plotados em gráficos da condutividade aparente versus distância, e 0 conjunto de perfis permitiu desenhar os mapas de condutividade aparente para cada profundidade teórica investigada, 7,5 e 15 metros através da interpolação dos dados entre as linhas através do software SURFER ${ }^{\circledR}$, versão 6 da Golden Software Inc. 0 método de interpolação utilizado foi a Krigagem linear entre pontos, que consiste num processo de estimativa por médias móveis de vaIores de variáveis distribuídas adjacentemente no espaço a partir de valores amostrados, ou seja, estima valores médios nos locais não amostrados a partir dos pontos amostrados. 0 raio de busca utilizado no método da Krigagem linear foi de 25 metros.

\section{Método GPR}

0 georadar, ou radar de penetração do solo (GPR - ground penetrating radar) é um método eletromagnético que emprega ondas de rádio em freqüências muito altas (normalmente entre 10 $1000 \mathrm{MHz}$ ) para localizar estruturas e feições geológicas rasas de subsuperfície ou localizar objetos enterrados pelo homem (Davis \& Annan, 1989; Annan \& Cosway, 1992).

Este método consiste na emissão de ondas eletromagnéticas (EM) e a recepção das ondas refletidas em interfaces no subsolo, produzindo como resultado uma imagem de alta resolução da subsuperfície. Os principais fatores que controlam o sinal do georadar são: a freqüência e a velocidade da onda EM no meio, 0 coeficiente de reflexão (contraste de permissividade dielétrica entre os meios) e a atenuação do meio. A atenuação é proporcional à condutividade elétrica do meio, de forma que quanto maior a condutividade maior a atenuação.

A técnica mais comum de aquisição de dados de georadar é a técnica de afastamento constante (common offset), onde um par de antenas (uma transmissora e outra receptora) é deslocado ao mesmo tempo ao longo de um perfil. Neste trabalho as aquisições com 0 georadar foram realizadas utilizando antenas não-blindadas de freqüência central de $200 \mathrm{MHz}$, no modo transversal elétrico (Annan \& Cosway, 1992). Os traços foram adquiridos a cada 0,25 m, com empilhamento de 128 sinais, digitali- 


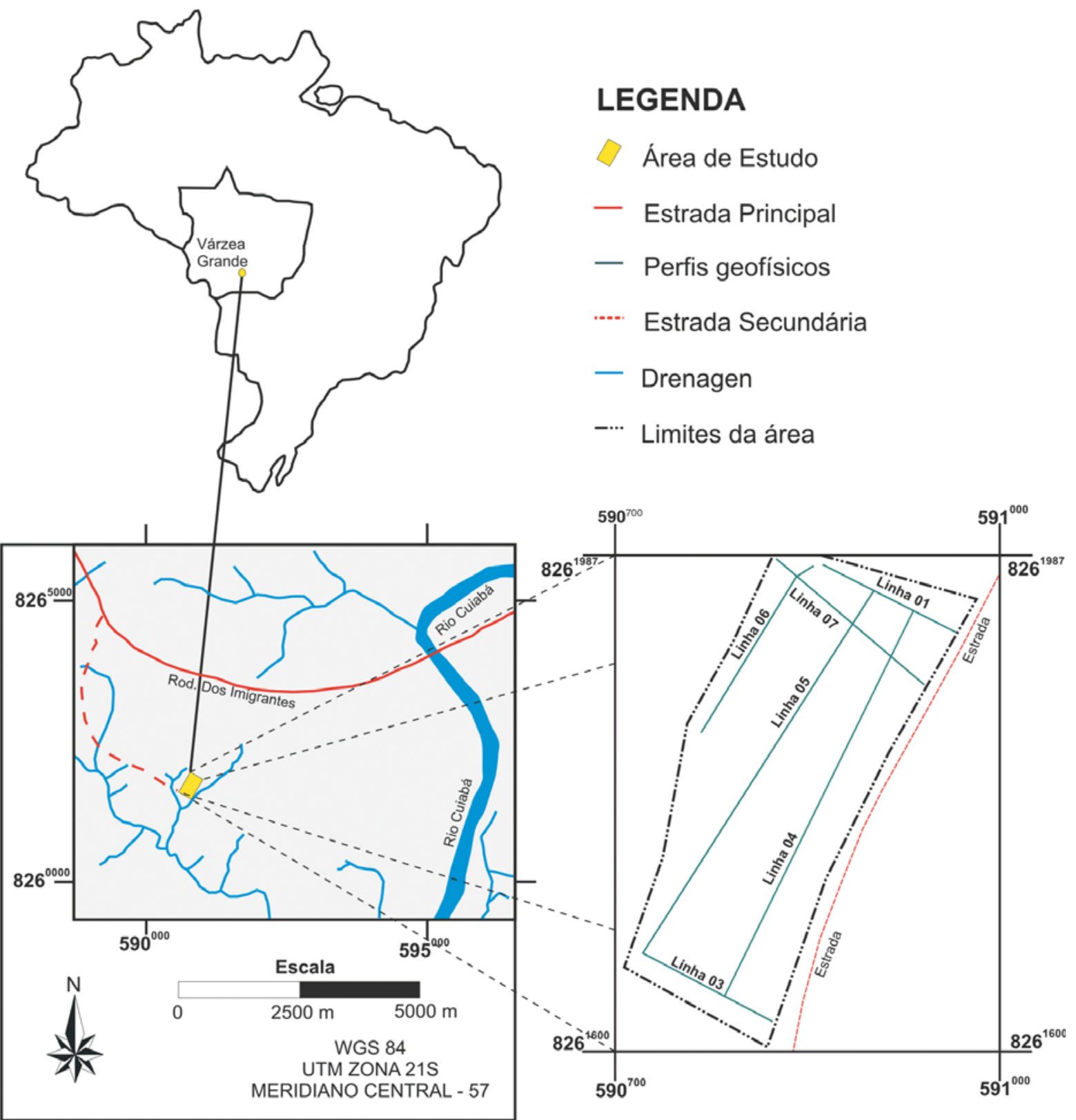

Figura 1 - Mapa de localização da área de estudo e dos perfis onde foram realizadas as investigações geofísicas.

zando 512 amostras por traço e uma freqüência de amostragem de $1847 \mathrm{MHz}$. Os perfis foram realizados sobre as linhas 4, 5, 6 e 7 (Figura 1). Os radargramas foram processados utilizandose 0 software ReflexW versão 3.5 (Sandmeier, 2004). A rotina de processamento consistiu em conversão do formato $*$.rd3 para $0 *$ dat, ajuste do tempo zero, filtros temporal (dewow e bandpass frequency) e ganhos (linear e exponencial). Os mesmos parâmetros de ganhos e filtros foram aplicados a todas as seções com 0 intuito de comparação das amplitudes dos sinais.

\section{RESULTADOS}

Antes de iniciar a apresentação dos resultados obtidos foi adotada uma classificação para os intervalos de resistividade elétrica $(\rho)$, condutividade elétrica aparente $\left(\sigma_{a}\right)$, e atenuação do sinal de GPR que sugestivamente identificam e separam as zonas contaminadas. Esta classificação foi elaborada conforme resultados geofísicos, apresentados em trabalhos, em áreas de disposição de resíduos sólidos e provenientes da indústria do couro no Bra- 
Tabela 1 - Classificação para as áreas contaminadas de acordo com intervalos de resistividade elétrica, condutividade elétrica aparente e atenuação da onda eletromagnética do GPR.

\begin{tabular}{|l|c|c|c|}
\hline \multicolumn{1}{|c|}{ Classificação } & $\sigma_{a}(\mathrm{mS} / \mathrm{m})$ & $\rho$ (ohm.m) & Atenuação no sinal do GPR \\
\hline AC - Área Contaminada & $>40$ & $<30$ & Alta \\
\hline AS - Área Suspeita de Contaminação & 20 a 40 & 30 a 50 & Média \\
\hline AN - Área Não Contaminada & $<40$ & $>50$ & Baixa \\
\hline
\end{tabular}

sil (Elis \& Zuquette, 1998, 2001; Elis, 1999; Shiraiwa et al., 2002; Moura, 2002; Lago, 2004; Pereira et al., 2004; Cunha, 2005) e os valores obtidos neste trabalho. Os intervalos adotados para esta classificação foram estabelecidos a partir dos valores de condutividade aparente coletados em campo e resultados de inversão de dados de resistividades aparentes obtidos nos caminhamentos elétricos, relacionando as áreas com contaminação comprovada, isto é, locais onde se encontram dispostas as valas e os resíduos em superfície. As áreas contaminadas (AC) apresentam condutividade elétrica aparente acima de $40 \mathrm{mS} / \mathrm{m}$, resistividades abaixo de 30 ohm.m e elevadas atenuações da onda eletromagnética do GPR. As áreas suspeitas de contaminação (AS) mostram valores de $\sigma_{a}$ entre 20 a $40 \mathrm{mS} / \mathrm{m}, \rho$ no intervalo de 30 a 40 ohm.m e atenuação GPR intermediária. Já as áreas não contaminadas (AN) mostram baixa atenuação da onda eletromagnética do GPR, condutividades elétricas abaixo de $40 \mathrm{mS} / \mathrm{m}$ e altas resistividades (acima de 50 ohm.m) (Tabela 1).

\section{Método da Eletrorresistividade}

As Figuras 2a, 2b e 2c mostram os resultados dos caminhamentos elétricos efetuados sobre as linhas 1,3 e 4 . A Figura 2a representa o modelo geolétrico obtido da Linha 3. Este perfil foi realizado em um local totalmente ausente de resíduos e deste modo este perfil mostra os valores médios (background) de resistividade dos metassedimentos do Grupo Cuiabá sem presença de contaminantes. No modelo geoelétrico observa-se que há a predominância de valores de resistividade no intervalo definido como área não contaminada (AN) (resistividades superiores a $50 \mathrm{ohm} . \mathrm{m}$ ), sendo percebidos 2 horizontes geoelétricos, um de alta resistividade elétrica com valores acima de 300 ohm.m e outro de baixa resistividade $(<200$ ohm.m). 0 primeiro entre 0 e $3 \mathrm{~m}$ de profundidade é interpretado como uma cobertura de solo concrecionário em conjunto com metadiamictitos alterados. A zona de baixa resistividade, que ocorre abaixo de 3 metros é relacionada ao aumento do teor de umidade devido à proximidade com a zona saturada. De acordo com dados de piezômetros instalados na área (Lannes, 2002), o nível d'água varia entre 4 e 6 metros de profundidade.
0 modelo geolétrico da Linha 1 (Figura 2b) mostra nitidamente duas zonas distintas, uma resistiva e outra condutiva, com um contato abrupto entre si. A primeira, localizada entre 0 e $50 \mathrm{~m}$, possui valores de resistividade no intervalo de 50 a 3000 ohm.m, sendo relacionada aos metadiamictitos da Formação Rio Coxipó. A zona condutora possui valores de resistividade elétrica inferiores a 50 ohm.m, se localiza entre 50 e $78 \mathrm{~m}$, sendo correlacionada a área suspeita de contaminação (AS) (valores entre $30 \mathrm{e}$ 50 ohm.m) e a área contaminada (AC) ( $\rho<30$ ohm.m).

Os resultados do caminhamento elétrico realizados sobre a Linha 4 (Figura 2c) mostram valores de resistividade que indicam ausência de contaminação (AN) nos primeiros 65 metros do perfil, após esta posição, notam-se quantidades consideráveis de resíduos depositados em superfície, influenciando nos valores de resistividade. No modelo geoelétrico obtido para a Linha 4 (Figura 2c) observa-se duas zonas de alta resistividade, que marcam 0 inicio e final do perfil, e uma de baixa resistividade. A primeira zona de alta resistividade aparece entre as posições 0 a $70 \mathrm{~m}$ da linha, relacionada aos metadiamictitos da Formação Rio Coxipó (AN). A segunda zona resistiva ocorre a partir da posição $70 \mathrm{~m}$ da linha, da superfície até a profundidade de 2,5 metros, relacionada ao solo concrecionário observado durante a aquisição de dados. Abaixo da profundidade de 2,5 metros ocorre uma zona condutiva de resistividade elétrica inferior a 50 ohm.m, correlacionada a área contaminada (AC) por resíduos sólidos do curtume.

\section{Método Eletromagnético Indutivo}

Os dados de condutividade obtidos em campo foram plotados em gráficos da condutividade aparente versus distância e o conjunto de perfis permitiu desenhar os mapas de condutividade aparente para cada profundidade teórica investigada de 7,5 e 15 metros (Figuras 3a e 3b) respectivamente através da interpolação de dados entre as linhas utilizando-se o método da krigagem. Os mapas de condutividade elétrica aparente para as duas profundidades apresentam comportamentos semelhantes, tendo aproximadamente as mesmas áreas anômalas.

A zona anômala, referente à área contaminada (AC), ocorre 

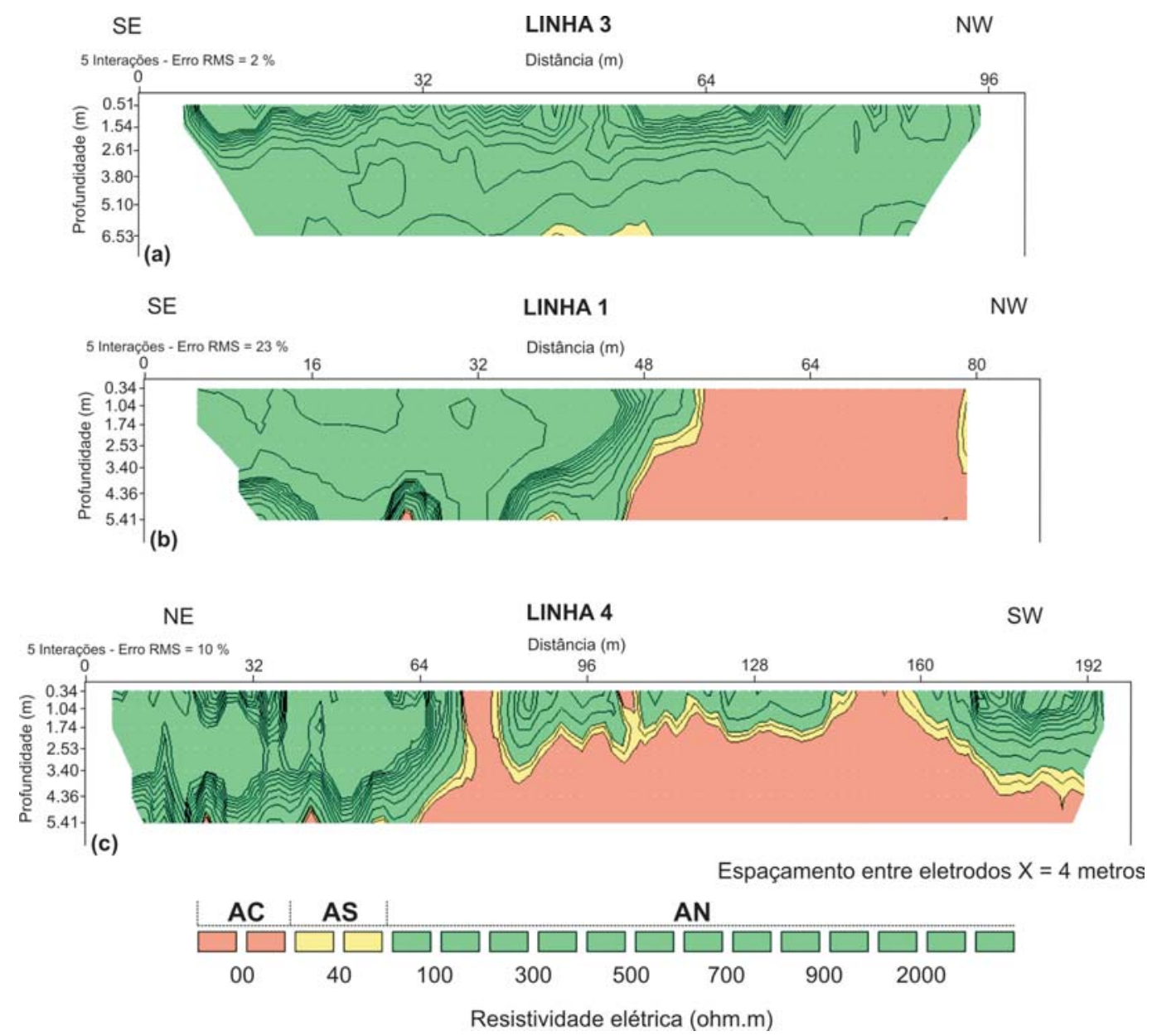

Figura 2 - Seções de resistividade elétrica 2-D: (a) Linha 3, (b) Linha 1, (c) Linha 4.

principalmente na parte centro norte da área (Figura 3a). Nesta região são visíveis, em alguns pontos, rejeitos expostos a céu aberto dentro das cavas e dispostos em superfície.

A área suspeita de contaminação (AS) é observada, em ambos os mapas, nas partes nordeste e centro leste dos mapas (Figura 3a e 3b). É caracterizada pela transição entre a AC e a AN.

A área não contaminada (AN) só aparece na parte sudoeste dos mapas (Figura 3a e 3b). É caracterizada pela ausência de resíduos sólidos industriais em subsuperfície, sendo correlacionada a metassedimentos do Grupo Cuiabá, não contaminados.

\section{Método GPR}

A Figura 4 mostra as seções de georadar que foram adquiridas sobre das Linhas 4, 5, 6 e 7. Uma faixa acima de cada perfil mostra com cores as zonas de elevada atenuação da onda eletromagnética (em cor-de-rosa), correlacionadas com as áreas contaminadas (AC), ao passo que as zonas de menor atenuação (em verde) estão correlacionadas com as áreas não contaminadas (AN) e mostram fortes reflexões a profundidades maiores. Assim é possível verificar áreas de contaminação na Linha 4 , entre as posições de $65 \mathrm{a} 145 \mathrm{~m}$, onde os sinais mais fortes do radar (em roxo na figura) ocorrem até 40 ns (tempo de ida e volta da onda eletromagnética). A partir de $165 \mathrm{~m}$ os sinais de radar aparecem até $80 \mathrm{~ns}$. Na Linha 5, a zona contaminada ocorre entre 160 e $225 \mathrm{~m}$. A partir de $225 \mathrm{~m}$ os sinais atingem até os 80 ns da zona não contaminada. As áreas contaminadas causam 0 efeito de zona de sombra nos dados de GPR, ou seja, impedem a propagação da onda eletromagnética devido à elevada condutividade dos resíduos sólidos e dos líquidos provenientes da percolação de água meteórica nestes resíduos.

Na Linha 6 a área não contaminada está entre as posições 70 e $120 \mathrm{~m}$ do perfil. Na linha 7 a zona contaminada está entre as posições 35 e $130 \mathrm{~m}$. 

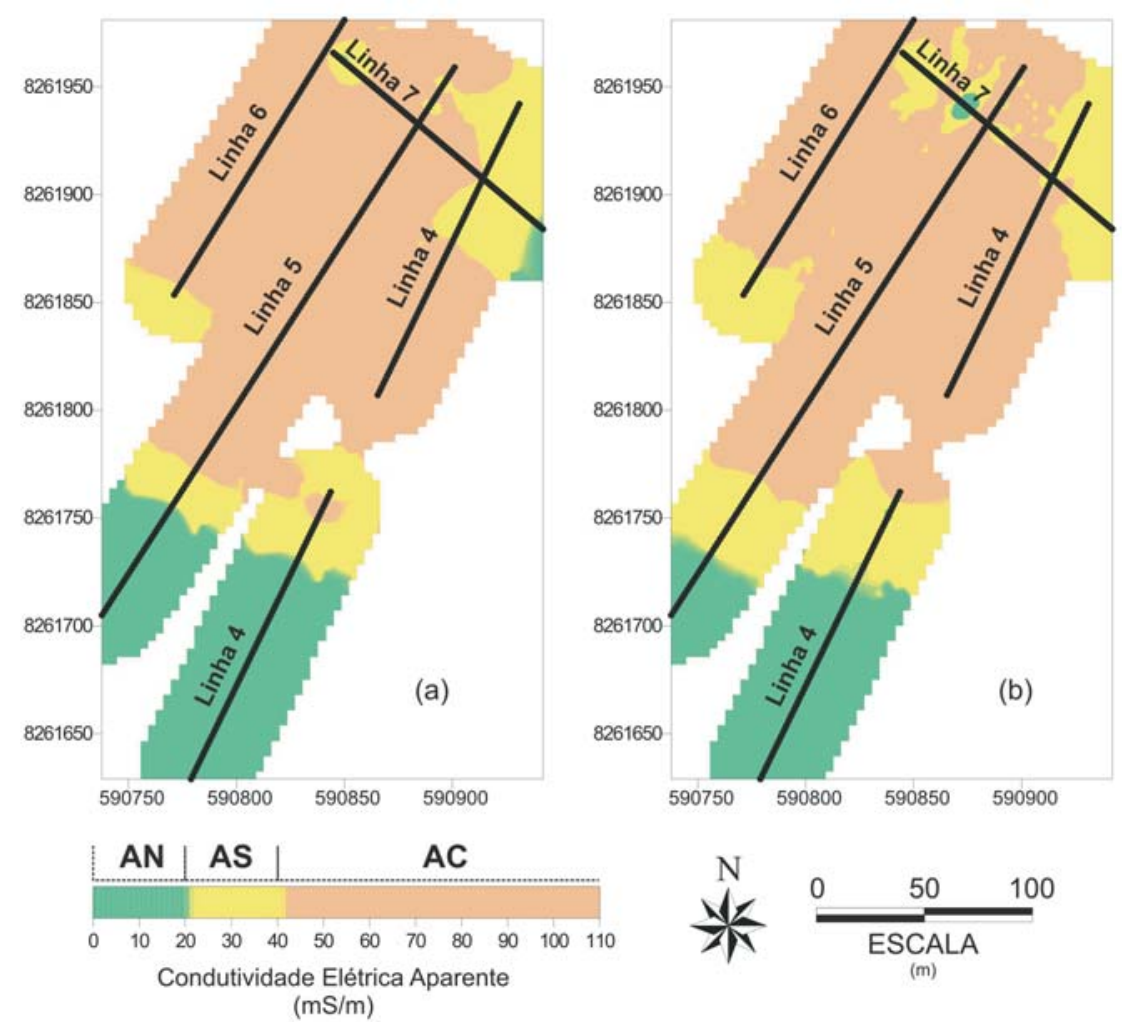

Figura 3 - Mapas de condutividade aparente da área: (a) Profundidade de 7,5 metros. (b) Profundidade de 15 metros.
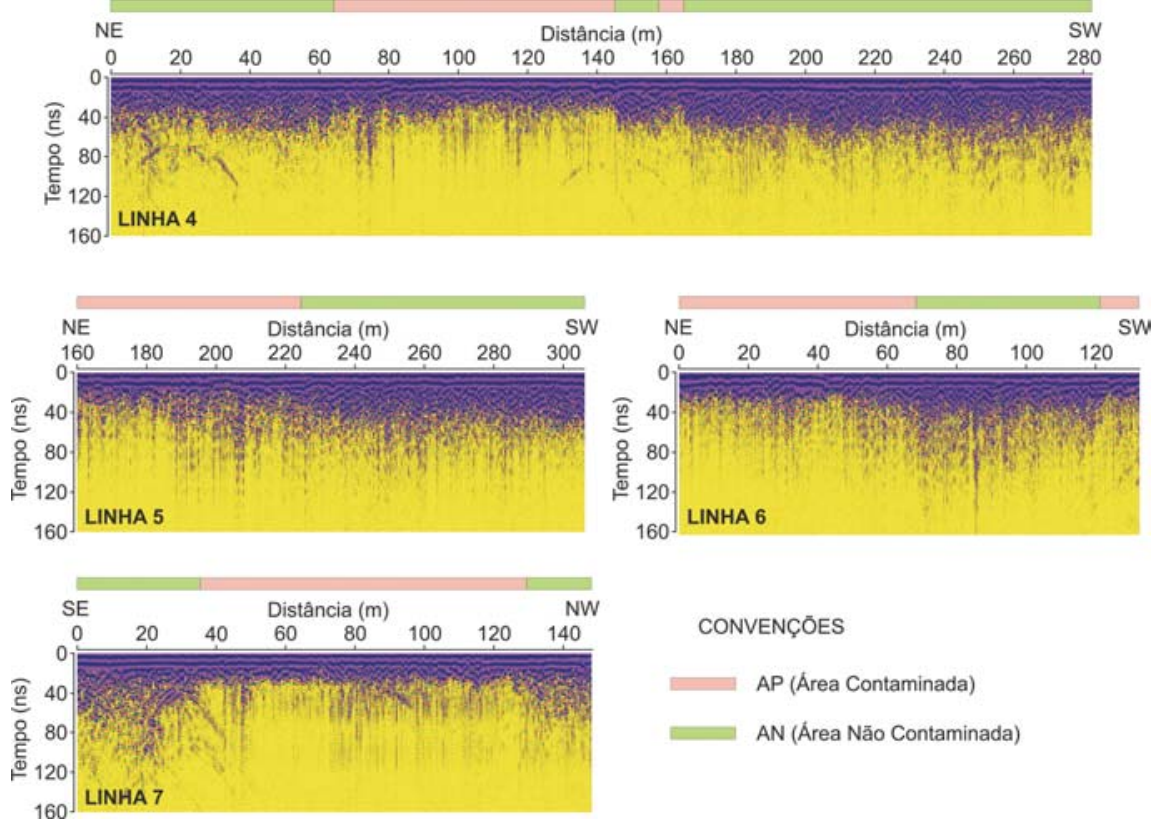

CONVENÇŌES

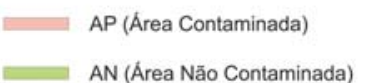

Figura 4 - Seções de georadar das linhas 4, 5,6 e 7. A faixa colorida acima de cada perfil indica as áreas contaminadas (rosa) e as áreas não contaminadas (verde). 


\section{CONCLUSÕES}

No geral os métodos geofísicos mostraram resultados relevantes, levando-se em conta os objetivos deste trabalho de localizar zonas contaminadas dentro de uma área de disposição de resíduos sólidos provenientes de um curtume.

Os mapas, de condutividade elétrica aparente, obtidos com o EM-34, nos modos DMH e DMV, através dos valores de condutividade, permitiram localizar e diferenciar as zonas AC (áreas contaminadas), AS (áreas suspeitas de contaminação) e AN (áreas não contaminadas), tanto ao longo das linhas como na distribuição na área levantada. A coincidência com a área utilizada pelo depósito é bem marcada. Não foi possível delinear a forma geométrica das cavas, pois o sistema eletromagnético utilizado fornece a condutividade elétrica média do volume entre as bobinas (Mcneill, 1980) atingindo pelo menos 7,5 $\mathrm{m}$ de profundidade e assim não fornece resolução para identificar alvos pequenos, ou seja, cavas de 2,5 a 4 metros. Um sistema com menor alcance poderá detectar estas cavas. Este método mostrou-ser 0 mais rápido e fácil de se executar em campo, permitindo levantar as cinco linhas com espaçamento entre os pontos de 1 a 2 metros.

Os resultados dos caminhamentos elétricos, realizadas com 0 arranjo dipolo-dipolo, mostraram ótimos resultados em seção transversal da linha, identificando claramente as zonas anômalas, tanto em extensão lateral, como em profundidade. A aquisição é a mais demorada, mas os resultados são mais detalhados.

0 georadar permitiu delinear com precisão os limites das zonas contaminadas e livres de contaminação, através dos contrastes de atenuação do sinal eletromagnético. 0 levantamento é rápido em terrenos limpos, livre de vegetação. A presença de obstáculos no terreno dificulta os levantamentos e introduz ruídos (antenas não-blindadas) nos sinais. Esperava-se verificar reflexões características das cavas, mas não foi identificado claramente nas seções.

\section{AGRADECIMENTOS}

Os autores agradecem à FAPEMAT (A Fundação de Amparo à Pesquisa do Estado de Mato Grosso) pelo apoio e financiamento da pesquisa do projeto "Estudo da disposição final de resíduos sólidos de curtume como fonte de contaminação em águas subterrâneas. Inovação tecnológica auxilia na preservação do meio Ambiente". Ao técnico Alexandrino Leite Nascimento, às geólogas Tânia H. Marcelino e Karen Crystyna 0. Gonçalves, ao geólogo Ivandro Schoenherr, ao Prof. Ms. Rodrigo Meireles Mattos Rodrigues e aos alunos da disciplina Prospecção Geofísica pelo auxílio na aquisição dos dados de campo. Aos reviso- res anônimos pelas críticas e sugestões que contribuíram para finalização deste artigo.

\section{REFERÊNCIAS}

ANNAN AP \& COSWAY SW. 1992. Ground penetrating radar survey design. In: Symposium on the Application of Geophysics to Engineering and Environmental Problems, 5ft, Oakbrook, Illinois. Proceedings..., Oakbrook, SAGEEP, 2: 329-352.

AQUINO VF. 2000. Métodos geofíisicos eletromagnéticos aplicados ao diagnóstico do solo e das águas subterrâneas em área de infiltração de resíduos industriais. Dissertação de mestrado, Instituto de Geociências, Universidade de São Paulo, São Paulo, 121 pp.

CETESB. COMPANHIA DE TECNOLOGIA DE SANEAMENTO AMBIENTAL. 2001. Manual de Gerenciamento de Áreas Contaminadas. Projeto de Cooperação Técnica Brasil-Alemanha/CETESB-GTZ. 2. ed., São Paulo, 398 pp.

CHERNICHARO CAL, SPERLING MV, SILVA PC \& GONÇALVES RF. 1996. Tratamento de efluentes de curtumes através de filtros anaeróbios seguidos por biofiltros aerados. In: Anais do XXV Congreso Interamericano de Ingenieria Sanitaria y Ambiental, Brasília, DF, 2: 715-724.

CUNHA LFJ da. 2005. Mapeamento da Pluma de Contaminação em Depósitos de Resíduos da Indústria do Couro, Através de Métodos Geofísicos - Dois casos em Mato Grosso. Dissertação de Mestrado em Física e Meio Ambiente, ICET/UFMT, Cuiabá - MT, 105 pp.

DAVIS JL \& ANNAN AP. 1989. Ground Penetrating Radar for High Resolution Mapping of oil and rock stratigraphy. Geophysical Prospecting. 37: $531-551$.

ELIS VR. 1999. Avaliação da aplicabilidade de métodos elétricos de prospecção geofísica no estudo de áreas utilizadas para disposição de resíduos. Tese de Doutorado, Instituto de Geociências e Ciências Exatas, UNESP, Campus de Rio Claro SP, 264 pp.

ELIS VR \& ZUQUETTE LV. 1998. Avaliação de contaminação por resíduos de curtume com a utilização de métodos elétricos. In: Anais do Encontro Regional de Geotecnica e Meio Ambiente, $2^{\circ}$ Workshop de Geofísica Aplicada, CD-ROM, Rio Claro - SP. ABGE.

ELIS VR \& ZUQUETTE LV. 2001. Aplicação Integrada de Métodos Geoeléctricos em Áreas de Disposiçãa de Resíduos Industriais: Cidade de Franca, Brasil., Geotecnia, 92: 47-68.

GIANNETTI BF, ALMEIDA CMVB, BONILLA SH \& VENDRAMET 0. 2001. Nosso Cromo de cada dia: Benefícios e Riscos. Revista de Graduação da Engenharia Química, Ano IV, 8: 55-58.

GOLDEN SOFTWARE, INC. 1995. Surfer ${ }^{\mathrm{TM}}$ for Windows V6. Reference Manual. Golden, Colorado, USA.

KELLER W, CHERNICHARO CAL, LAUFFER J \& MOREIRA RV. 1997. 0 cromo nos resíduos sólidos de curtumes - Imobilização em materiais 
cerâmicos. In: Anais do 19th Congresso Brasileiro de Engenharia Sanitária e Ambiental, Foz do Iguaçu - PR, 1: 1759-1768.

LANNES S. 2002. Avaliação da qualidade da água subterrânea na área de disposição do lodo proveniente do curtume Berto S/A-Várzea Grande/MT. Instituto de Saúde Coletiva (Dissertação de Mestrado), UFMT, CuiabáMT, $100 \mathrm{pp}$.

LAGO AL. 2004. Aplicação integrada de métodos geofísicos em área de disposição de resíduos sólidos urbanos em Bauru-SP, Dissertação de Mestrado, Instituto de Astronomia, Geofísica e Ciências Atmosféricas USP, São Paulo/SP, 110 pp.

LIMA OAL de, SATO HK \& PORSANI MJ. 1995. Imaging industrial contaminant plumes with resistivity techniques. Journal of Applied Geophysics, 34: 93-108.

LOKE MH. 2004. Res2Dinv ver. 3.53 for Windows 98/Me/2000/NT/XP. Rapid 2D Resistivity \& IP Inversion using the least-squares method. Software Manual, $129 \mathrm{pp}$.

McNEILL JD. 1980. Electromagnetics terrain conductivity measurement at low induction numbers. Tech. Note TN-6, Geonics Ltd, Mississauga, Ontario, Canada, 13 pp.

MIGLIORINI RB. 1999. Hidrologia em Meio Urbano. Tese de Doutorado. Instituto de Geociências. Universidade de São Paulo. 146 pp.

MOURA HP. 2002. Emprego da eletrorresistividade e da polarização induzida na caracterização geoelétrica de áreas de disposição de resíduos sólidos urbanos. Tese de Doutorado, Instituto de Geociências e Ciências Exatas, UNESP, Campus de Rio Claro, SP, 231 pp.

PAPP JF. 1994. Chromium life cycle study. Information Circular 9411 , Bureau of Mines, United States Department of the Interior, 102 pp.
PEREIRA PA, REGO MJM do \& LIMA OAL de. 2004. Diagnóstico da contaminação hídrica subterrânea e superficial provocada por resíduos sólidos urbanos e de curtume, no município de Alagoinhas, BA. In: Anais do I Simpósio de Geofísica da SBGf. CD-ROM, São Paulo SP, 4 p.

PORSANI JL, FILHO WM, ELIS VR, SHIMELES F, DOURADO JC \& MOURA HP. 2004. The use of GPR and VES in delineating a contamination plume in a landfill site: a case study in SE Brazil. Journal of Applied Geophysics, 55(3): 199-209.

REYNOLDS JM. 1997. An introduction to Applied and Environmental Geophysics. John Wiley \& Sons, New York NY, USA, 796 p.

SANDMEIER KJ. 2004. REFLEXW Version 3.5, Windows 9x/2000/NT/XP. Program for the processing of seismic, acoustic or electromagnetic reflection, refraction and transmission data. Software Manual, 192 pp.

SHIRAIWA S, LISOVSKY SP, ELIS VR, PORSANI JL \& BORGES WR. 2002. Estudos geofísicos integrados no lixão de Cuiabá, MT, Brasil Resultados Preliminares. Revista Brasileira de Geofísica - SBGf, 20(3): 181-186.

U.S. ENVIRONMENTAL PROTECTION AGENCY. 1997. Technology Alternatives for the Remediation of Soils Contaminated with $\mathrm{As}, \mathrm{Cd}, \mathrm{Cr}$, $\mathrm{Hg}$, and $\mathrm{Pb}$. Engineering Bulletin. EPA/540/S-97/50.

WARD OSH. 1990. Resistivity and Induced Polarization Methods. Geotechnical and Environmental Geophysics, Volume 1: Review and Tutorial. Society of Exploration Geophysics, 147-189.

\section{NOTAS SOBRE OS AUTORES}

Sergio Junior da Silva Fachin. Bacharel em Geologia pelo Instituto de Ciências Exatas e da Terra da Universidade Federal de Mato Grosso em 2005. Integrante do Núcleo de Geofísica e Estudos Ambientais da UFMT entre os anos de 2001 a 2005, participando de projetos em Geofísica Aplicada (Métodos elétricos, eletromagnéticos aplicados à Geologia, Geotecnia, Hidrogeologia e Meio Ambiente). Atualmente Mestrando em Geofísica Aplicada pelo Instituto de Astronomia, Geofísica e Ciências Atmosféricas da Universidade de São Paulo, desenvolvendo o projeto intitulado "Mapeamento Geoelétrico Temporal 2D no Antigo Lixão de Ribeirão Preto-SP".

Felix Huber. Bacharel em Geologia pelo Instituto de Ciências Exatas e da Terra da Universidade Federal de Mato Grosso em 2005. Integrante do Núcleo de Geofísica e Estudos Ambientais da UFMT entre os anos de 2002 a 2005, participando de projetos em Geofísica Aplicada (Métodos elétricos, eletromagnéticos aplicados à Geologia, Meio Ambiente, Geotecnia e Hidrogeologia). Atualmente presta serviços de geologia nas áreas de Mineração, Hidrogeologia, Geofísica Aplicada e Meio Ambiente.

Shozo Shiraiwa. Concluiu o doutorado em Geofísica pela Universidade de São Paulo em 1994. Professor de Física Geral e Prospecção Geofísica da Universidade Federal de Mato Grosso, publicou 3 artigos em periódicos especializados e 56 trabalhos em anais de eventos. Possui 4 itens de produção técnica. Orientou 4 dissertações de mestrado, além de ter orientado 16 trabalhos de iniciação científica e 7 trabalhos de conclusão de curso nas áreas de Geociências, Agronomia e Engenharia Elétrica. Entre 1997 e 2003 participou de 5 projetos de pesquisa, sendo que coordenou 2 destes. Atualmente participa de 5 projetos de pesquisa. Atua na área de Geociências, com ênfase em Geofísica Aplicada a problemas ambientais. Em suas atividades profissionais interagiu com 121 colaboradores em co-autorias de trabalhos científicos utilizando métodos gravimétricos, magnéticos, eletrorresistividade, eletromagnéticos e radar de penetração no solo.

Welitom Rodrigues Borges. Geólogo pelo Instituto de Ciências Exatas e da Terra da Universidade Federal de Mato Grosso (ICET/UFMT) em 2000. Em 2002 obteve 0 título de Mestrado em Ciências, área de Geofísica Aplicada, pelo Instituto de Astronomia, Geofísica e Ciências Atmosféricas da Universidade de São Paulo (IAG/USP). Atualmente está fazendo o Doutorado (bolsista Fapesp 02/07510-0) junto ao Programa de Pós-Graduação em Geofísica do IAG/USP, desenvolvendo a pesquisa "Caracterização geofísica de alvos rasos com aplicações no planejamento urbano, meio ambiente e arqueologia: Estudo sobre o sítio controlado do IAG/USP", e trabalha com processamento de dados sísmicos e eletromagnéticos no Laboratório de Geofísica Computacional (LGC/IMEC) da Unicamp-Campinas. 
Renato Blat Migliorini. Geólogo pela Universidade de Fortaleza (1985). Mestre (1994) e Doutor (2000) em Geociências pelo Departamento de Recursos Minerais e Hidrogeologia do IGc/USP. Desde 1992 é Docente dos Departamentos de Geologia Geral e Engenharia Sanitária e Ambiental da Universidade Federal de Mato Grosso. Atualmente é chefe do Departamento de Geologia Geral da UFMT. 